\title{
Low dose effects research in Europe: eight years of evolution towards new paradigms
}

\author{
J. Repussard* \\ Former Director-General of IRSN and Chairman of MELODI, IRSN, 92262 Fontenay-aux-Roses, France.
}

Received: 27 September 2017 / Accepted: 27 September 2017

\begin{abstract}
In 2008, the question was raised at European level of how low dose ionizing radiation health effects, and the biological mechanisms behind them, could be better understood through research. This would allow enhancing radiation protection, particularly in the medical practice of radiation, and in managing people, economic production and the environment after a contamination accident. This article tells the story of the efforts in this direction made by the scientific community across Europe, encouraged by the evolution of EURATOM research policy. It describes the achievements, the encountered difficulties of all kinds, scientific, cultural and technocratic and looks ahead to the future.
\end{abstract}

Keywords: ionizing radiations / low dose effects / radiation protection sciences / EURATOM research / European platforms / strategic research agendas

\section{Introduction}

At the end of the first decade of the new century, and before the occurrence of the Fukushima disaster, some questions were starting to be voiced, on both sides of the Atlantic, about the added value of the "low dose effects" research activities, which appeared to be characterised by a lack of convincing results that could be practically be used to consolidate the international radiation protection system.

This issue is nevertheless critical since the vast majority of exposures to ionizing radiation are in the low dose domain (medical exposures, radon and environmental exposures after accident) with a large variety of dose rates and sequences involving a repetition of exposures. The "low dose effects" issue has however long been controversial, for several reasons:

- there is biological evidence of genotoxicity of ionising radiation at low dose, e.g., $1 \mathrm{mGy}$. In the last 15 years, progress of radiobiology has led to a gain of sensitivity of a factor 100 in such investigations (Rothkamm and Lobrich, 2003). This has led to the commonly accepted concept that the health effects of radiation exposure are without threshold, inducing fear in society;

- however, there is also evidence that the pathway to radioinduced cancer is complex, and requires a succession of biological mechanisms resulting from a combination of misrepaired DNA strands (Hanahan and Weinberg, 2011). There is also evidence that the efficiency of DNA repair mechanisms are influenced by individual genetic and

*Corresponding author: jrepussard@aol.com epigenetic characters, which to date remain largely unidentified (Weinberg, 2013);

- until recently, other health effects of exposure were believed to occur only above the range of "low doses". Recent research has however shown that such thresholds could be much lower than previously thought. A good example of this evolution of knowledge is the considerable work done on the issue of eye lenses exposure for workers, particularly some health professionals (ICRP, 2012). In the domain of medical exposures, there is also evidence that some late toxicity effects of radiotherapy on the cardio-vascular system are still not fully identified and understood. It is one of the goals of the new EURATOM MEDIRAD (MEDIRAD, 2017) project to investigate this important issue;

- epidemiology studies are important to demonstrate the health effects of low doses, and to justify radiation protection standards. So far some studies show an association of an excess of cancer exposures to IR at doses as low as $20 \mathrm{mGy}$ (Leuraud et al., 2017) although they are unable to establish a link of causality (Grosche, 2017). On the whole, however, there remains considerable uncertainty about the evidence of effects below $100 \mathrm{mGy}$, even though large efforts have been dedicated to increasing the size of cohorts, through international cooperation (Richardson et al., 2015). The development of cohorts informed by the collection of individual biomarker data should allow some further progress in the future (Bourguignon et al., 2017; Hall et al., 2017). This will probably require the development of cohorts in the medical domain. This again is one of the objectives of MEDIRAD to test such approach. 
In this context, the adequacy of current radiation protection protocols for low dose and low dose rate exposures remains a matter of controversy, not to mention the subject of hormesis, for which there is also evidence, such as the known anti-inflammatory effects of low dose exposure for example, and which might play a role in the DNA repair mechanisms, activated by natural radiation background exposure. Ten years ago, EURATOM decided to investigate the need for a novel strategy to approach these research questions, considering the high societal and economic impact of these unresolved issues, clearly shown through the debates on the actual health consequences of the Chernobyl and Fukushima accidents for example. This article tells the story of the remarkable efforts made by the scientific community in Europe towards an integrated development (across disciplines and countries) of research on the biological mechanisms which are set in motion by low-dose ionizing radiation, and on resulting health effects.

\section{New impetus with the 2009 European "High level and expert group" report}

The HLEG (High level expert group) was an initiative of representatives of national authorities (with a policy interest or substantial research activities in low dose risk research), launched within the EURATOM research programme with the active support of the European Commission (EC). The HLEG brought together in a unique configuration senior scientific experts and senior managers of research institutions from five European countries.

The objectives of the HLEG were:

- to formulate and agree the policy goals to be addressed by low dose risk research;

- to develop a strategic research agenda and road map for low dose risk research in Europe;

- to specify the essential elements of and next steps for establishing a sustainable operational framework for low dose risk research in Europe.

After a public consultation the HLEG published its final report in January 2009 (HLEG, 2009). In this report the HLEG recommended to set up a new European research Platform named MELODI, for Multidisciplinary European Low Dose Initiative, with a high ambition for the years to come:

"MELODI will aim, with a view to sustainable integration, to:

- bring together the programmes of the various funding bodies and research organisations in Europe;

- establish effective interfaces with stakeholders and the broader scientific and health community in Europe and beyond;

- ensure the availability of key infrastructures;

- establish an integrated approach for training and education, including knowledge management.

Increasingly rapid advances in biological and medical knowledge are providing new opportunities to achieve these goals."

In 2009, on the occasion of the first international symposium organised by ICRP to discuss scientific and other issues with its key stakeholders, the proposals made in the report of the HLEG were presented. This was received with polite interest. However, several senior US experts appeared to be somewhat puzzled by this European initiative: what more could be done on this subject, which has been studied in depth for decades with no sparing of resources, both in the frame of the USA/Japan research initiative related to the A-Bomb survivors cohort, and though numerous and varied experiments of radiobiology involving animal models in particular? All this work, with significant input from European countries, has served to develop the current ICRP doctrine, based on the hypothesis of a linear no-threshold dose/effect relationship for the increase of cancer risk at intermediate and low doses, with numerous correction factors taking into account the relative effects on different tissues, or of different qualities of radiation. This elaborated framework proved to be quite sufficient to effectively regulate radiation protection, so why look elsewhere? As it turned out, these views have since then prevailed in the USA, and the US DOE (Department of Energy) decided in 2015-2016 to withdraw the remaining budgets dedicated to low dose effects research.

But Europe had a different background for discussing that same topic: the questions raised by the Chernobyl accident were still resonating in a number of EU Member states, and in some, the future of nuclear energy was being publicly questioned. Thus the question of the public health impact of low dose radiation exposures was perceived as an issue to which research had not yet brought a sufficiently satisfactory answer, and which it was considered important to resolve.

Two sets of decisions were taken in the months following the publication of the HLEG report.

Within EURATOM, the European Commission proposed to the EURATOM Member states to fund a "network of excellence" in order to provide financial support for the follow up developments suggested by the HLEG report. Following this call of proposal, published in accordance with the 7 th EURATOM research program rules, research organisations in Europe proposed to form the "DoReMi" network of excellence, gathering 12 European research organisations. Coordinated by STUK, the nuclear and radiological safety authority in Finland, DoReMi was launched in January 2010 for a period of 6 years, with a budget of $13 \mathrm{M} €$ dedicated not to operate large research projects, but, in an innovative way, to scientific work aimed at the development of consensus on the approaches that should be preferred to address the issues of low dose effects and related risks in future European research programs. In the course of its operations, DoReMi launched several calls to associate further organisations (universities, research centers) to its work. Eventually 37 organisations were involved in DoReMi, which was later evaluated as one of the most successful Networks of Excellence of the 7th EURATOM work program.

In line with the HLEG recommendations, a group of 15 European research organisations decided, also in 2010 , to create MELODI as an independent association, governed by French law. The goal was to develop a perennial structure which would act as a European platform for the longer term coordination of research strategies in the field of low dose effects and risks, with a multidisciplinary approach. MELODI acts independently from the succession of EURATOM 


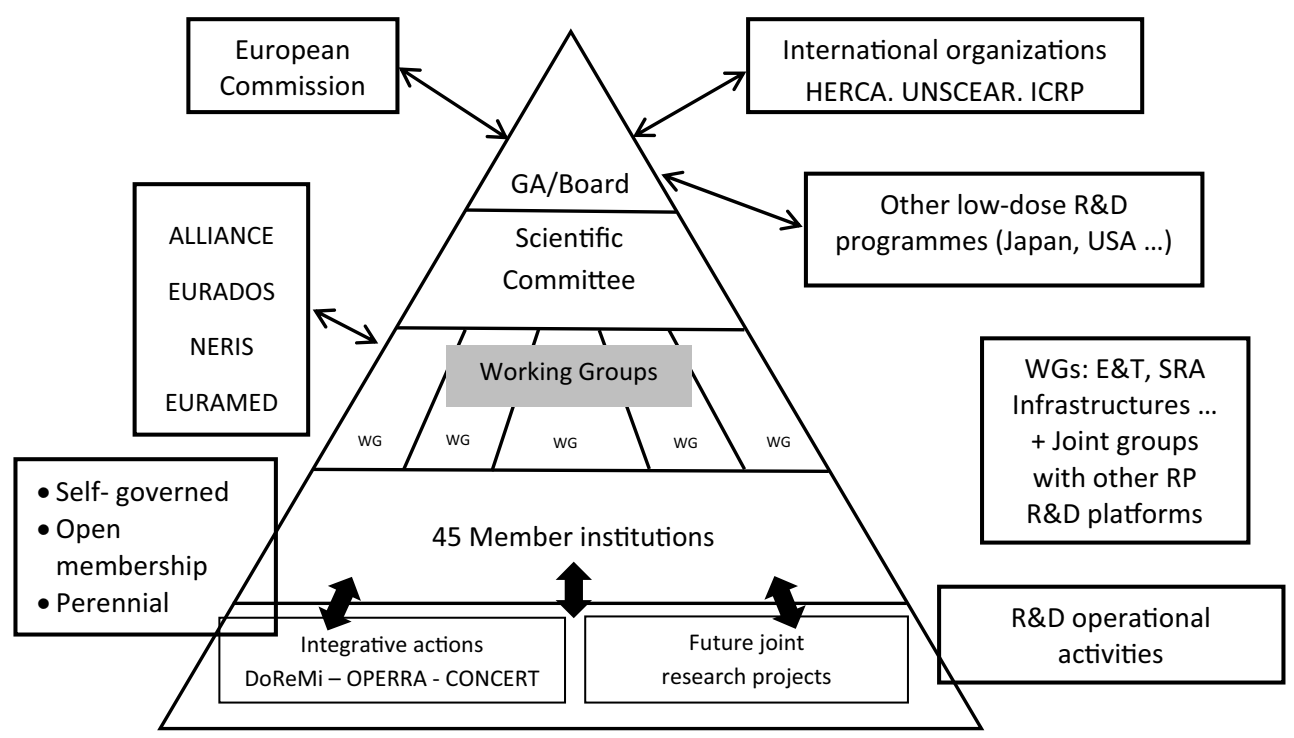

Fig. 1. Structure of the MELODI association.

projects, and therefore offers a supporting structure to develop research strategies which is open to all partners wishing to have membership, and which benefits from a horizon for its action not limited to the duration of EURATOM projects or constrained by EURATOM rules. Today, MELODI enjoys the participation of 45 research institutions from most EU countries, which are national research bodies, universities or medical associations.

\section{MELODI operations}

\subsection{A new research governance concept}

As explained above, the innovative approach of MELODI is to associate the scientific community members, through their respective institutions, to participate in the development of research strategies that will influence the EURATOM and national research policy orientations in a bottom-up and multidisciplinary problem based approach. The structure of MELODI is shown in Figure 1. It includes several instances, from the governing structure (General Assembly of members, Board, Bureau) to scientific groups of experts from the different disciplines of interest for research in the field of radiation protection, designated by member bodies (by election or consensus based decisions) and dedicated to key statutory issues: development of the Strategic research Agenda (SRA), education and training, research infrastructures, Scientific overseeing committee, etc. MELODI is not structurally funded by the EC, but by the statutory contributions of its members. However, some of the key tasks of MELODI are also referred to in specific EURATOM funded projects, and as such this work is partly funded by the EC. The good representativeness of this system was illustrated during one of the MELODI open Workshops in 2000 when a proposal for a list of ten priority research subjects, to be chosen from a long list of topics included in the MELODI strategic research agenda, was drawn up on one hand within MELODI Board (whose members are elected by the General Assembly), and on the other hand, through a direct vote open to all workshop participants (coming from all MELODI members and beyond): the list of ten priority subjects was the same in both processes held independently.

\subsection{A scientific ambition to respond to societal expectations}

The main mission of MELODI (MELODI SRA, 2016) has been published on its web site (www.melodi-online.eu) in several successive versions, gradually more synthetic as time passed and consensus developed within the association. However, the original thinking from the HLEG report and its list of "overarching questions" from society remained discernable: in a nutshell, MELODI SRA presents a matrix approach, where the different pathways for a type of effects (shape of dose-response for cancer; tissue sensitivities for cancer induction; individual variability in cancer risk; effects of radiation quality [type of radiation, dose rate]; risks from internal radiation exposure; risks of, and dose response relationships for, non-cancer diseases and hereditary effects) are to be researched on several scales: molecular, cellular, tissue, organ or function, whole organism, population.

\section{The progressive structuration of European research for radiation protection in sister platforms}

Soon after the creation of MELODI, complementary initiatives were taken in the adjacent - and more appliedfields of scientific investigations: environmental issues, with the setting up of the ALLIANCE ${ }^{1}$ (European Radioecology

\footnotetext{
${ }^{1}$ www.er-alliance.eu.
} 
Alliance) platform, emergency preparedness issues with NERIS $^{2}$ (European Platform on Preparedness for Nuclear and Radiological Emergency Response and Recovery), dosimetry, with the consolidation of the pre-existing EURADOS $^{3}$ (European Radiation Dosimetry Group) network into a platform, and lately medical exposure issues, with the creation in 2016 of EURAMED $^{4}$ (European Alliance for Medical Radiation Protection Research). The existence of this set of coordinated research platforms, in which some of the member bodies are identical (ensuring coherence), allows a representation of a variety of scientific disciplines and applicative areas of radiation protection research, and as such are valuable to the European Union. They are closely coordinated, mostly with a lead from MELODI, through Memoranda of Understanding (MoUs) signed between the platforms, which were extended to the main European medical organizations (ESR, EANM, ESTRO, EFRS, EFOMP) as an initiative to support the creation of a medical platform (EURAMED). This platform coordination, which is also encouraged by the transversal nature of EURATOM projects like OPERRA ${ }^{5}$ and CONCERT ${ }^{6}$ which cover the whole field of radiation protection research, allows the production of consolidated policy approaches and documents relevant to the whole field of radiation protection research, and facilitates multidisciplinary exchange between researchers.

To develop such exchange, MELODI initiated yearly open workshops where the developing strategic agenda and research priorities were discussed in depth. From 2016 this open dialogue approach was extended to all platforms, in the shape of a new concept: the European Radiation Protection Research Week (ERPW), which brought together in Oxford (UK) around three hundred participants. The second edition of this event, hosted by IRSN is taking place in Paris, in October 2017, and is combined with the fourth International Symposium of ICRP. This combined event ICRP-ERPW 2017 gathers over 500 participants from Europe and many countries worldwide.

The inclusion of social sciences and humanities in this landscape has also been discussed in recent years, lately in the frame of the RICOMET conferences which gather researchers and stakeholders in this field. The creation of yet another platform was suggested by some experts, whilst others, not contesting the need for coordinated research in this area also in the context of European radiation protection research, expressed concern about the feasibility of such an additional radiation protection research structure which would be separate from other platforms which also need an input from social sciences into their own development. Considering this rather narrow field of action (radiation protection) by comparison to the breadth of issues of societal nature concerning the broader scopes of energy or medical policies in Europe, which stakeholders are certainly eager to address also through scientific approaches, the format (within the broader disciplinary field of social sciences and humanities) and viability of the dialogue with other scientific communities

\footnotetext{
${ }^{2}$ www.eu-neris.net.

${ }^{3}$ www.eurados.org.

${ }^{4}$ http://www.eibir.org/scientific-activities/joint-initiatives/europeanalliance-for-medical-radiation-protection-research-euramed.

${ }^{5}$ www.melodi-online.eu/operra.html.

${ }^{6}$ www.concert-h2020.eu/.
}

and stakeholders in the other platforms should be discussed in depth.

\section{The evolution of EURATOM approach for radiation protection research}

\subsection{The concept of "European integration"}

Following the publication of the HLEG Report, EURATOM decided to shift some resources towards "integration" objectives, rather than to continue to fund research calls made of a collection of items recommended by Member states, which allowed to spend the budgets but not to reach satisfactory answers on major radiation protection issues. This new "integration policy" was a strong signal to the scientific communities to start working together in the framework of the new "platforms" on the definition and implementation of innovative strategic research approaches to the key remaining questions about the scientific basis for radiation protection. This policy was expressed in the form of successive calls leading to the setting up of transverse projects of a new kind (Fig. 2): DoReMi Network of Excellence, followed by OPERRA, and lately CONCERT EJP. DoReMi paved the way for the setting up of the first MELODI SRA, a task which was extended to a broader multiplatform field through OPERRA and CONCERT, with in particular the undertaking of two open Research and Technological Development (RTD) calls organized by the project, to initiate the implementation of priority research in line with the SRA's. Training and education initiatives for researchers were also initiated by these projects, also through dedicated calls.

The goals of this integration policy, pursued by the conjunction of the platforms actions and transversal EURATOM projects are rather ambitious, in line with the conclusions of the HLEG report:

- to gradually align the national research programs of EURATOM Member states on common priorities, optimizing the use of existing national and European resources; - to be able to address complex issues, as described in the SRA's, through well designed multi annual research actions which could surpass the limits of the smaller separate projects hitherto allowed by the successive EURATOM calls and national programs. For example, the discovery and validation of biomarkers for individual response to radiation exposure is a very complex task, which cannot be completed in a short time, or by a small subset of research organizations, even if prestigious: it requires in depth, open and probably lengthy discussions of initial experimental results, in particular to define commonly agreed validation protocols which will determine the field of validity and inherent limits for each candidate biomarker, and then to agree on controlled protocols to test their operational use. Potentially, the platforms only can make this happen, with the support of appropriate research projects channeling the necessary funds, because of the need to prepare and follow up research itself by collective discussions to prepare the next steps and optimize scientific strategies. This is in fact the concept of "Roadmaps", which the platforms are invited to design as a tool to implement as efficiently as possible the objectives of their SRA's; 


\begin{tabular}{|c|c|c|c|c|c|}
\hline Platforms & \multirow{2}{*}{ MELODI } & ALLIANCE & NERIS & EURADOS & EURAMED \\
\hline Projects & & & & & \\
DoReMi & & & & \\
$31 / 01 / 2010-$ & & & & \\
\hline Comet & & & & & \\
$01 / 06 / 2013-$ & & & & \\
$31 / 05 / 2017$ & & & & & \\
\hline Prepare & & & & & \\
$01 / 02 / 2013-$ & & & & \\
$31 / 01 / 2016$ & & & & \\
\hline OPERRA & & & & & \\
$01 / 06 / 2013-$ & & & & \\
$31 / 05 / 2017$ & & & & \\
\hline Concert EJP & & & & \\
$01 / 06 / 2015-$ & & & & \\
$31 / 05 / 2020$ & & & & \\
\hline MEDIRAD & & & & \\
$01 / 06 / 2017-$ & & & & \\
$31 / 05 / 2021$ & & & & & \\
\hline
\end{tabular}

Fig. 2. The EURATOM integration concept: platforms + projects $(80 \mathrm{M} €)$.

- to position the European Commission and EURATOM Member states authorities as regulators of research, defining strategic objectives, budgets and rules for excellence and competitiveness rather than trying to achieve these goals by running themselves the "nitty gritty process" of RTD yearly calls.

\subsection{A new financial instrument to support integration: the European Joint Program (EJP)}

One of the key challenges on the road to integration is the development of synergies between national and EURATOM research programs. In 2014, it was decided by EC and Member states to make use for radiation protection research of a new "co-fund" instrument, only used so far for the Fusion program, the European Joint Program (EJP). This is based on a sharing of costs between EURATOM (70\%) and National funding from the participating countries $(30 \%)$, for a total foreseen expenditure of $27 \mathrm{M} €$ over 5 years, of which $60 \%$ will be dedicated to the funding of open calls along the priorities of the platforms SRA's, the rest being for integration projects $(30 \%)$ and for administration (10\%). The EURATOM call led to the acceptance in 2015 of the CONCERT project, coordinated by the Bundesamt für Strahlenschutz (BfS) in Germany, in which 28 organizations from 22 EU Member states, as well as the four European platforms are participating as Consortium members with voting rights. However, the rules attached to the EJP instrument foresee that the $\mathrm{EC}$ will reimburse a maximum of $70 \%$ of all eligible expenditure by the CONCERT Consortium, within the budget limit. The $30 \%$ remaining part has to be borne by the Consortium, implicitly with the support of national research programs. A difficulty may therefore appear when the open RTD calls operated by CONCERT lead to fund projects in part operated by organizations outside the Consortium: while an unwritten but consensual rule is that each country is responsible for funding its share of the $30 \%$ cofund, the members of the consortium may not be in a position to operate a cash transfer to third parties from their country which have won a call, such a transfer being the only way to establish the eligibility of reimbursement by the EC. This difficulty has led to a perception of complexity and sometimes unfairness of the whole process, especially as the Consortium members, and their legally affiliated organizations are able to justify their own full expenditure for CONCERT in accordance with auditable financial data recording their activity (time sheets, etc.). There is therefore an urgent need to improve the rules for such co-fund instruments for the future, in order to provide equal opportunities to all member states institutions who want to take part in future open research calls on such a co-fund basis. In June 2017, the platforms sent a formal letter to the European Commission to draw attention on this important issue, together with a detailed note framing some proposals for the future European EURATOM research program. These documents are available on MELODI website in particular.

\section{The necessary continuation of the international scientific dialogue}

Obviously, while Europe has spent significant resources in establishing new research approaches to resolve pending issues affecting the radiation protection system of ICRP, those same issues continue to be discussed in multilateral institutions such as UNSCEAR, ICRP, WHO, IAEA, OECD/NEA. Multinational research projects, such as those which aim to consolidate international nuclear worker cohorts bringing together European countries, USA, Canada for example, continue to play a lead role in maintaining and developing international research efforts. It would be very useful to set up a scientific forum where not only questions are debated, but also where research strategies and "roadmaps" could be discussed, bringing a new focused approach which could be shared across Europe, America and Asia. An international seminar invited in the fall of 2016 by the Electric Power 


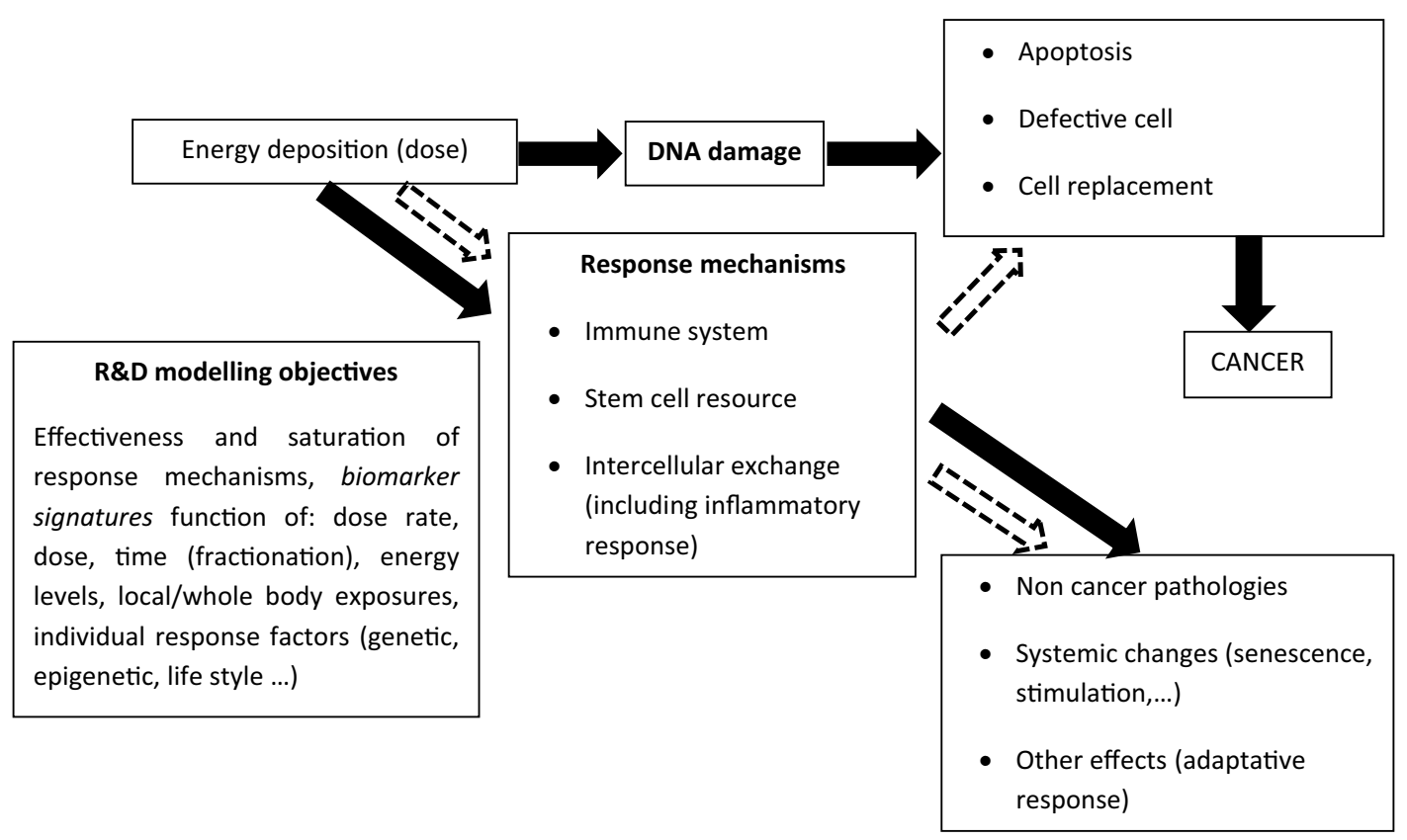

Fig. 3. Research challenges regarding low dose/dose rate radiation effects: a Strategic Research Agenda approach; dotted arrows show the biological effects of low dose deposition which are not sufficiently investigated and modeled to support operational radiation protection protocols.

Research Institute (EPRI, 2017) in USA did illustrate a potential for a consensus on such a vision between the participants.

\section{The way forward past H2020: critical factors of a successful integration of European research for radiation protection}

The past decade, which spanned two EURATOM research programs known as FP7 (2007-2013) and Horizon 2020 (2014-2020), has allowed the inception of an ambitious perspective of "integration". As for many other European policies, it remains indispensable to keep in mind the reasons why this policy was decided, to overcome the remaining difficulties that are in the way of progress, in the minds of people or actual administrative issues that need to be resolved, and to evaluate and communicate results to all stakeholders concerned, as well as to the public through appropriate media.

Some of the scientific challenges are much too complex for any single organization or short term European consortium to hope to resolve them on its own. A typical example is the question of understanding the mechanisms behind varying individual response to ionizing radiation, which requires the establishment of a carefully planned road map of the successive research steps which need to be covered to come to validated and peer reviewed operational tools useable for instance by radiotherapists. Time must be spent by key experts to design and agree on such road maps, which usually involve different disciplines, and complementary sources of information. Although these scientists will eventually compete in open calls for the implementation of parts of the roadmap, their investment in the initial planning step is a key success factor for all, and it is the role of the platforms to harbor such precompetition collective thinking. This has started over the recent years, even if for some researchers this activity is seen as a EC imposed burden slowing down laboratory research rather than an opportunity to plan carefully for their future projects.

Figure 3 illustrates the complexity of the research challenges which still lie beneath the words "low dose/dose rate radiation effects". And yet overcoming these challenges actually governs the capability to improve the justification of medical exposures, and the optimal management of "unplanned exposures" caused by actual or potential accidental radioactive releases in the environment, which are the two major societal concerns related to non-professional exposures to ionizing radiation. Beyond the general objective of radiation protection principle of optimization, aiming to reduce exposure to a level "as low as reasonably achievable" (a must for planned professional exposures), the objective is to provide trustable tools ("trustable" by the health professionals, the competent regulatory authorities, and the public) allowing to establish the relative health risk due to the exposure, in the light of the other health risks resulting from alternative risk management decisions: risks to the patient resulting from relying in non-ionizing radiation diagnostic/therapies (failure of the diagnostic or therapy); risks to the population resulting indirectly (depression, suicide...) from long term evacuation decisions aiming at avoiding continued "existing" exposures, to use ICRP vocabulary, for instance after an accidental radioactive contamination of the environment.

Going back to the fundamental conclusions of the HLEG group a decade ago, continued success in overcoming these major research challenges will require perseverance in the two directions which have been outlined in recent EURATOM policies:

- the continued and even reinforced implication of researchers in the discussion and consensus forming around research roadmaps and strategic agendas. This needs to be 


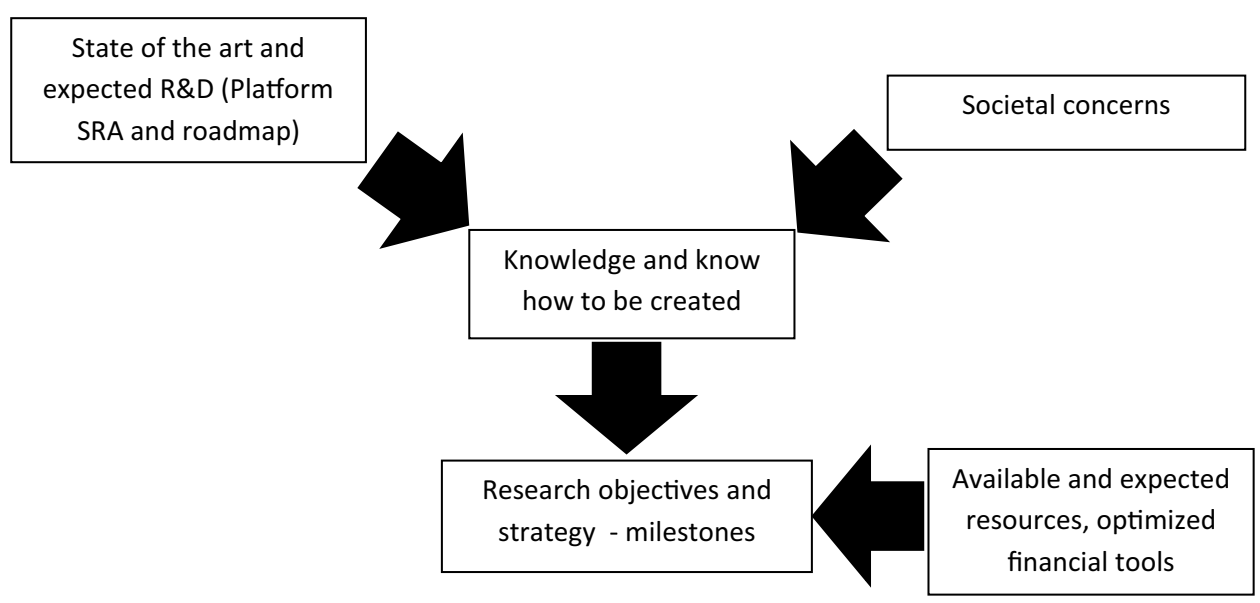

An ambitious project in the scope of CONCERT which suppose an open dialogue among the scientific communities and with the stakeholders and the public authorities to move forward from a "laboratory logic" to an "European integrated logic"

Fig. 4. The need for joint research roadmaps.

strongly encouraged by the management of national research organizations, by providing time and supporting resources for this, as well as by recognizing the importance of networking and of adhering to joint European research strategies and shared projects;

- the continued and even reinforced support by Member states and the European Commission to a European integration policy which has started to produce results. This needs in particular to resolve the problem of combining safely, legally speaking, in a single European funding instrument the two complementary concepts of cofunded research partnerships, and of open competitive research calls to implement agreed research roadmaps (Fig. 4). Co-funding is essential for full integration of scarce resources confronted to complex and expensive research challenges, and to plan research strategies in a concerted approach at European level, involving the research community with a medium to long term outlook. Open competitive calls are essential to draw excellence, and to attract university research capabilities from beyond the traditional EURATOM scientific circles, particularly from the medical area, but also other different sectors such as physics or ecology, or social sciences. Such a combination could require a revision of the existing cofund instrument, or the development of an ad-hoc approach for radiation protection research, which would then need to be approved by all EURATOM Member states. Such an adhoc solution has been adopted for example in the field of metrology research, where a consortium of the national metrology institutes (called EMPIR) was successfully formed to operate jointly European research, reaching out where necessary to outside research capabilities. This initiative was however facilitated by the rules of procedure of the general European research framework, which do not require unanimity of Member states as do the EURATOM rules.

\section{Conclusion}

Three main lessons may be drawn from the experience gained over the last eight years of discussing research strategies on low dose effects in Europe:

- the potential operational consequences in terms of radiation protection and risk perception of a better understanding of these effects are major. They would significantly affect the evolution of medical practice of the ionizing radiation resource, for imaging and therapy. They would allow a more optimal management of unplanned population exposures following accidental releases of radioactivity in the environment, and possibly a better understanding of actual radiological risks by the populations concerned. For professional exposures, which often involve highly fractionated doses at medium ranges of dose rates, new knowledge on biological effects might lead to review the practice of a dose/dose rate coefficient (DDERF), in favor of a "new coefficient" to ponder effects more effectively;

- important break-through and validated results can only be expected to occur as a consequence of approaching the problems through new research paradigms. These include a much closer scientific cooperation between "medical" and "nuclear" research (biology and radio-biology; development of patients cohorts where exposure to ionizing radiation is a precisely documented parameter among other biological and pathological records). They also include the acceptance of following a collective roadmap for research, which provides the continuity and medium term engagement allowing projects standard steps (thesis for example) to become meaningful in a broader perspective design. They also include, because of this longer term perspective, the recognition that work on "effects", in terms of biology, does not need to be immediately connected to drawing conclusions on "radiological risks" or related pathologies: 
this segmentation, foreseen by agreed roadmaps, would lead to new sets of research publications demonstrating "effects" of low dose/low dose rate exposures, to be later examined (by the same teams, or by other teams) in terms of validation of relevant biomarkers for given exposure spectrums, and then in terms of exploitation of these biomarkers, for medical or radiation protection purposes. For this to happen, it is however necessary to significantly improve the European funding instruments, to consolidate the European platforms, and the national links between all concerned institutions;

- a consequence of the above, but this may be the most difficult change to produce, is the necessary recognition that no single research team, laboratory, institution or even country can hope to resolve on its own, in a fully convincing way, the still mysterious issues of low dose/low dose rate effects, which are an intrinsic part of life on planet earth. This recognition is necessary for the collective efforts of strategy conception to be seen not just as an exercise drawing resources away from research, but as a precondition for any successful research enterprise in this domain, a collective investment useful for every laboratory in Europe and beyond.

\section{References}

Bourguignon M, Bérard P, Bertho JM, Farah J, Mercat C. 2017. What's next in Radioprotection? Radioprotection 52(1): 21-28.

EPRI International Dose Effect Alliance, November 2016 Workshop Proceedings, EPRI 2017 Technical Report. Available from http:// radiationeffects.org/wp-content/uploads/2017/03/IDEA-Conf-Re port-11.2016.pdf.
Grosche B. 2017. Editorial comment on: Mortality in the French cohort of nuclear workers, Radioprotection 52(3): 211-212.

Hall $\mathrm{J}$ et al. 2017. Ionizing radiation biomarkers in epidemiological studies - An update, Mutat. Res. 771: 59-84.

Hanahan D, Weinberg RA. 2011. Hallmarks of cancer: the next generation, Cell 144: 646-674.

HLEG (High Level and Expert Group) report. European low dose risk research radiation protection. 2009. EUR 23884 ERC. Available from http://cordis.europa.eu/pub/fp7/fission/docs/hleg_re port 2009_en.pdf.

ICRP, 2012. ICRP statement on tissue reactions/early and late effects of radiation in normal tissues and organs - Threshold doses for tissue reactions in a radiation protection context. ICRP Publication 118. Ann. ICRP 41(1/2).

ICRP-ERPW 2017 congress web site. Available from http://www. icrp-erpw2017.com.

Leuraud K, Fournier L, Samson E, Caër-Lorho S, Laurier D. 2017. Mortality in the French cohort of nuclear workers, Radioprotection 52(3): 199-210.

MEDIRAD. 2017. Implications of medical low dose radiation exposure. European Horizon 2020 Project. Available from http:// cordis.europa.eu/project/rcn/211042_en.html.

MELODI Strategic Research Agenda (SRA). 2016. Available from http://www.melodi-online.eu/doc/MELODI_SRA_2016_final. pdf.

Richardson DB et al. 2015. Risk of cancer from occupational exposure to ionising radiation: retrospective cohort study of workers in France, the United Kingdom, and the United States (INWORKS), BMJ 351: h5359.

Rothkamm K, Lobrich M. 2003. Evidence of a lack of DNA double strand break repair in human cells exposed to very-low X-ray doses, PNAS 100(9): 5057-5062.

Weinberg RA. 2013. The Biology of Cancer. New York USA: Garland Science.

Cite this article as: Repussard J. 2017. Low dose effects research in Europe: eight years of evolution towards new paradigms. Radioprotection 52(4): 251-258 